In the opening chapter Gabbiana and Rungger-Brändle discuss the fibroblast, the fundamental cell in repair. From work in human beings and experimental animals, this cell has been shown to be remarkably adaptable and may alter its morphological, biochemical and functional features. In wound healing, fibroblasts, and possibly other cells, transform into contractile cells called myelofibroblasts and these are probably concerned in wound contraction. Unfortunately, the authors cannot yet identify the factors controlling this process. Fibroblasts are also involved in the synthesis and metabolism of connective tissue, including collagen. Collagen has several functions as well as its primary role as a support. Duance and Bailey describe the five known types of collagen but admit that the relationship between structure and function is unknown. As many as 15 cell types may be involved in wound healing, including cells derived from the bone marrow, thymus, reticuloendothelial system and liver, as well as the cells of the tissues involved.

In regeneration, growth-regulating factors encourage the tissue to replace damaged areas with normal-functioning cells. Usually the formation of scar tissue is preceded by physical or other trauma, but this is not always the case; for example, in Dupuytren's contracture and scleroderma trauma is absent.

The book is nicely balanced between a consideration of basic processes and how these apply in the skin, central nervous system, lungs, heart and kidney. The editor himself has written an excellent chapter on the pathology of scar tissue, which links several of the authors' contributions. All authors agree that hyperplasia and atrophy, regeneration and repair are extremely complex phenomena, but in this book they have generally succeeded in making the subject intelligible to their readers.

Each chapter includes between 200 and 500 references which indicates the coverage of the subject. It also probably accounts for the fact that there are few references after 1978, although in one chapter additional notes have been added in 1980 .

I can recommend this excellent book; it will be very useful for medical and nonmedical scientists in many disciplines. Considering the complexity of the subject, the book is easy to read - presumably the result of sound editing - and there are few mistakes of any note and little overlap between contributions. Further volumes on the immunology of inflammation and the molecular basis for anti-inflammatory therapy will round off the series, which, when complete, should prove a most valuable addition to medical libraries in particular.

N.R. Rowell is Reader in Dermatology at the University of Leeds, and Senior Consultant Physician in the Department of Dermatology, Leeds General Infirmary.

\title{
Modelling the growth of literature
}

\section{B.C. Vickery}

Scientific Information Systems and the Principle of Selectivity. By W. Goffman and K.S. Warren. Pp.191. ISBN 0-03-056081-0. (Praeger/Holt-Saunders: 1980.) £13.75, $\$ 19.95$.

IMPRESSED by the ever-growing rate of scientific publication, the authors of this book decided that the process of scientific communication needed to be studied as an "ecosystem", They develop a mathematical model for the growth of the literature in a defined subject, and then go on to a detailed quantitative analysis of various bodies of literature. The main experimental data consist of a bibliography of schistosomiasis, covering the period 1852-1962 and comprising over 10,000 references. There is little use made of the mathematical model in subsequent analysis, and there is no convincing demonstration of its validity.

Six kinds of analysis were made: distribution of articles and authors by language and by date; multiple publication by individual authors; multiple authorship of articles; most prolific journal titles and distribution of articles among journals; similar analyses for sub-areas of schistosomiasis research; and movement of researchers among sub-areas. The most interesting of the analyses is that based on co-authorship of articles. If authors $A$ and B collaborate, also B and C, as well as C and $D$, then $A, B, C, D$ can be said to form a network. Of the 6,500 authors in the schistosomiasis bibliography, 2,300 had no collaborators, but there was one big network that embraced 1,750 authors, as well as several hundred smaller networks.

This network of co-authorship is the only real evidence of scientific communication that is examined in the book. The citation of one author by another (implying awareness and probable reading of another's work) is not explored, still less any other forms of communication (attendance at conferences, for example).

The authors then conducted a qualitative evaluation of the schistosomiasis literature. Some 47 active workers in this subject were chosen with the aid of expert advisers. Each was asked to examine the bibliography and indicate "articles you deem of lasting importance", Over 3,000 of the 10,000 articles were selected at least once; about 1,600 at least twice; 471 at least five times; and 69 papers were selected 12 times or more. As one moved from the 3,000 to the 69 , the likelihood of the authors being members of the large coauthorship network not surprisingly increased from 40 per cent to 70 per cent.

Goffman and Warren are convinced that "quality filtering" of scientific literature is needed in order to ease the task of retrieval, and offer proposals based on citation in review papers as an indicator of quality. I find it doubtful whether any further mechanism - other than the review papers themselves - is needed by the scientific community.

The book presents a number of interesting ideas about the structure of scientific literature, and Goffman and Warren certainly extract as much as they can from the data used. But this information simply consists of the authors, title and source of each article, plus its evaluation by experts. There is no indication of what the authors have read, or who reads their contribution. In order to get a more rounded understanding of scientific communication, the book needs to be complemented by the reading of work such as that of Garvey, who has taken these other factors into consideration.

Brian Vickery is Director of the School of Library Archive and Information Studies, University College London.

\section{The complete angler}

\section{Bruce R. Moore}

Circular Statistics in Biology. By Edward Batschelet. Pp.372. ISBN 0-12-081050-6. (Academic: 1981.) £28.80, \$69.50.

MANY scientists who rarely otherwise cite secondary sources, do so routinely when referring to statistical tests. Several reasons for this tradition are evident, not the least of which is that original statistics papers are meant to be read by statisticians.

This use of secondary sources is frequently quite harmless, since clear and accurate statistics texts are available at many levels. It has sometimes been less satisfactory, however, for that substantial minority of scientists whose data occur in vector form. The $t$-tests and ANOVAs of everyday statistics are seldom applicable to angular data. A number of special tests have, in fact, been developed, but they are not described in most statistics texts and are therefore missed by many potential users. In consequence, statistical inference based upon mere intuition has become the norm in certain areas.

Against this background, one finds isolated regions where the special tests have been thoroughly exploited. The source most of ten cited in these areas is an out-ofprint monograph by Edward Batschelet (American Institute of Biological Sciences, 1965). Although extremely specialized, this work has become for many readers not only a highly trusted source but even an object of personal affection. Such persons were pleased to learn in recent years that, while approaching retirement, Professor 